\title{
Mapping the evolving landscape of super- enhancers during cell differentiation
}

\author{
Yan Kai ${ }^{1 \dagger}$, Bin E. Li ${ }^{2 \dagger}$, Ming Zhu ${ }^{3 \dagger}$, Grace Y. Li ${ }^{2}$, Fei Chen ${ }^{3}$, Yingli Han ${ }^{3}$, Hye Ji Cha ${ }^{2}$, Stuart H. Orkin ${ }^{2,4}$, Wenqing Cai ${ }^{2 *}$,
} Jialiang Huang ${ }^{3^{*}}$ and Guo-Cheng Yuan ${ }^{1,5^{*}}$ (I)

\author{
* Correspondence: caiwqwq@gmail. \\ com; jhuang@xmu.edu.cn; guo- \\ cheng.yuan@mssm.edu \\ ${ }^{+}$Yan Kai, Bin E. Li and Ming Zhu \\ contributed equally to this work. \\ ${ }^{2}$ Cancer and Blood Disorders \\ Center, Boston Children's Hospital \\ and Dana-Farber Cancer Institute, \\ Harvard Medical School, Boston, MA \\ 02115, USA \\ ${ }^{3}$ State Key Laboratory of Cellular \\ Stress Biology, Innovation Center for \\ Cell Signaling Network, School of \\ Life Sciences, Xiamen University, \\ Xiamen 361102, Fujian, China \\ 'Department of Pediatric Oncology, \\ Dana-Farber Cancer Institute and \\ Harvard Medical School, Boston, MA \\ 02115, USA \\ Full list of author information is \\ available at the end of the article
}

\begin{abstract}
Background: Super-enhancers are clusters of enhancer elements that play critical roles in the maintenance of cell identity. Current investigations on super-enhancers are centered on the established ones in static cell types. How super-enhancers are established during cell differentiation remains obscure.

Results: Here, by developing an unbiased approach to systematically analyze the evolving landscape of super-enhancers during cell differentiation in multiple lineages, we discover a general trend where super-enhancers emerge through three distinct temporal patterns: conserved, temporally hierarchical, and de novo. The three types of super-enhancers differ further in association patterns in target gene expression, functional enrichment, and 3D chromatin organization, suggesting they may represent distinct structural and functional subtypes. Furthermore, we dissect the enhancer repertoire within temporally hierarchical super-enhancers, and find enhancers that emerge at early and late stages are enriched with distinct transcription factors, suggesting that the temporal order of establishment of elements within super-enhancers may be directed by underlying DNA sequence. CRISPR-mediated deletion of individual enhancers in differentiated cells shows that both the early- and late-emerged enhancers are indispensable for target gene expression, while in undifferentiated cells early enhancers are involved in the regulation of target genes.
\end{abstract}

Conclusions: In summary, our analysis highlights the heterogeneity of the superenhancer population and provides new insights to enhancer functions within superenhancers.

Keywords: Super-enhancers, Enhancer, Differentiation, Dynamics, 3D genome, Hierarchy

(c) The Author(s). 2021 Open Access This article is licensed under a Creative Commons Attribution 4.0 International License, which permits use, sharing, adaptation, distribution and reproduction in any medium or format, as long as you give appropriate credit to the original author(s) and the source, provide a link to the Creative Commons licence, and indicate if changes were made. The images or other third party material in this article are included in the article's Creative Commons licence, unless indicated otherwise in a credit line to the material. If material is not included in the article's Creative Commons licence and your intended use is not permitted by statutory regulation or exceeds the permitted use, you will need to obtain permission directly from the copyright holder. To view a copy of this licence, visit http://creativecommons.org/licenses/by/4.0/. The Creative Commons Public Domain Dedication waiver (http://creativecommons.org/publicdomain/zero/1.0/) applies to the data made available in this article, unless otherwise stated in a credit line to the data. 


\section{Background}

Enhancers are distal regulatory elements critical to establishing and maintaining cell type-specific gene expression programs. Dense clusters of enhancers, known as stretch enhancers or super-enhancers (SEs), are occupied by high density of master regulators, cohesins, mediators, and coactivators and are decorated with unusually high H3K27ac signals [1-4]. SEs are thought to play an important role in the maintenance of cell identity, and disruption of their activities, such as by genetic mutation, has been linked to higher susceptibility to diseases $[2,4,5]$. Recent studies suggest that a subset of SEs contain hierarchical structure [6-10]. Within a hierarchical SE, the hub enhancer plays a critical role in orchestrating its structural and functional organization [7].

It remains poorly understood how SEs are established temporally during cell differentiation or in response to an environmental cue. Previous studies are sporadic and limited in scope [11-14]. These analyses suggest that entire SEs may be established rapidly to initiate inflammatory response [11]. A few case studies have dissected the evolving SE landscape during differentiation in a limited number of loci. For example, two studies $[10,15]$ examined the progressing landscape of one SE near the Wap locus during mammary gland's differentiation and found that there is temporal and functional hierarchy between the elements. Despite these advances, a systematic understanding of the dynamic establishment of the SE landscape during cell differentiation is still lacking.

To investigate the general patterns of SE establishment during cell differentiation, here we developed a systematic approach to map the evolving landscape of SEs during cell differentiation based on the dynamic pattern of chromatin states. We applied this method to several publicly available time-course chromatin immunoprecipitation followed by sequencing (ChIP-seq) data (Fig. 1a). Our analysis identified three distinct SE subtypes, each associated with a distinct temporal pattern. We integrated RNA-seq and Gene Ontology (GO) information to gain functional insights and interrogated $\mathrm{Hi}-\mathrm{C}$ data to dissect the features associated with higher-order genome organization. We further applied CRISPR/Cas9 genome-editing assays to dissect the functionality of different constituent elements within temporally hierarchical SEs. We found that both the early- and late-emerged enhancers during differentiation are indispensable for target gene expression in differentiated cells. In summary, our analyses highlight the heterogeneity of the SE population and provide new insights into the dynamic establishment of SEs during cell differentiation.

\section{Results}

Mapping the evolving landscape of super-enhancers during differentiation reveals three distinct patterns

To map the evolving landscape of SEs in differentiated cells, we developed an unbiased clustering approach to classify SEs according to temporal histone modification patterns during cell differentiation. We focused on the H3K27ac mark, which is the most wellcharacterized histone mark associated with enhancer activities and often used as the basis to identify SEs [1]. To this end, we first identified constituent elements by calling H3K27ac peaks using MACS1.4 [16] and then identified SEs by using the ROSE program [1]. The temporal pattern of H3K27ac signals associated with each SE can be quantified by a numerical vector, where each element corresponds to the H3K27ac 


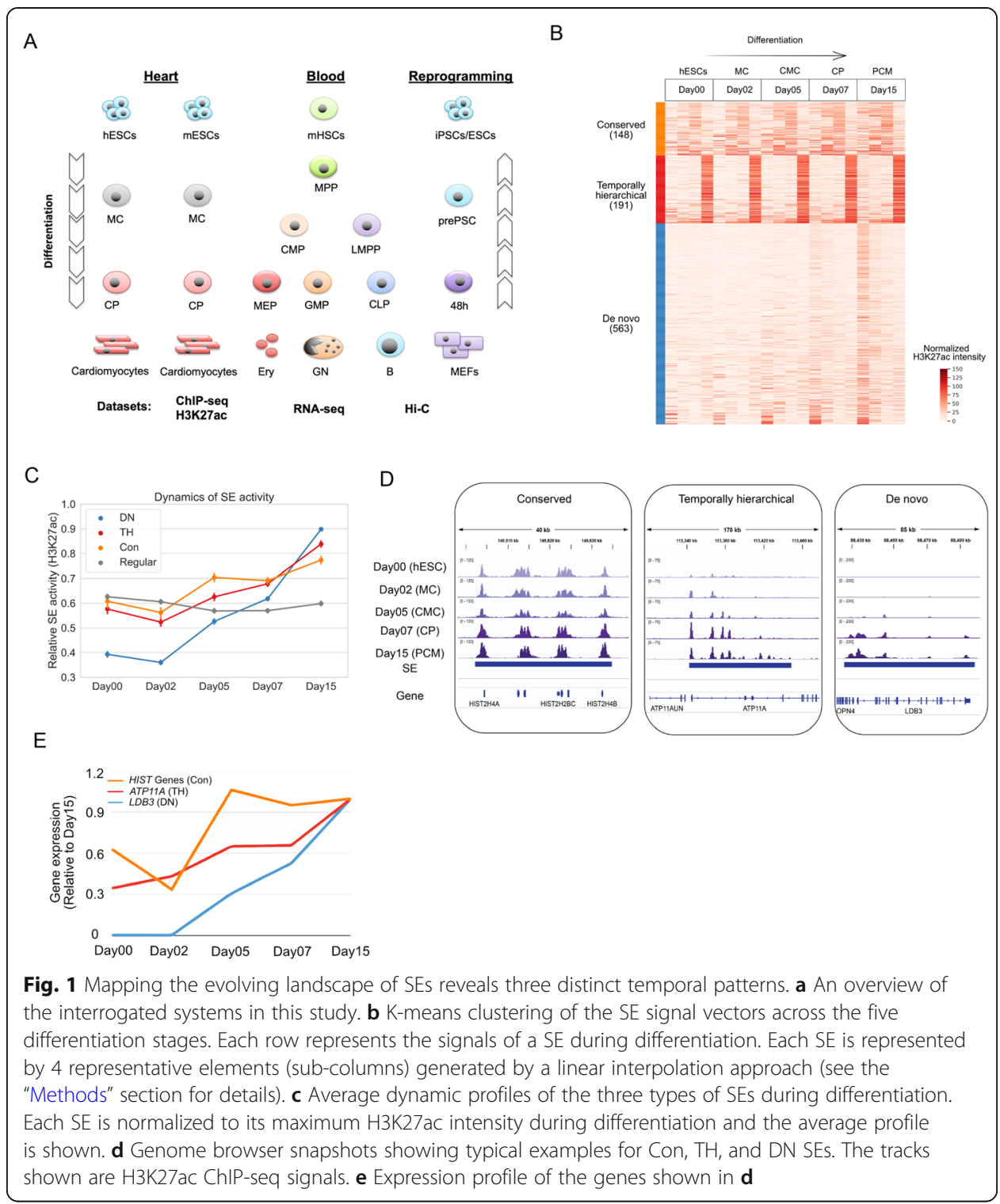

intensity of a constituent element at a time point. The dimensionality of these vectors varies from one SE to another because the number of constituent elements is not a constant (Additional file 1: Figure S1a), which leads to differential dimensions for clustering. To overcome this challenge, we standardized the vector dimension by using a linear interpolation approach (see Methods for details, Additional file 1: Figure S1b). We then applied k-means clustering $(K=3)$ to divide SEs into distinct groups based on the Euclidean distance between the standardized vectors. We validated this classification approach by using various parameters and obtained similar results, suggesting that this method is robust to the choice of different parameter values (Additional file 1: Figure S1c-d, see Methods for details).

We first applied this approach to dissect the evolving landscape of SEs during human cardiomyocyte differentiation, by using a public ChIP-seq dataset [17]. In this study, the authors carried out ChIP-seq experiments to profile the dynamic changes of genome-wide H3K27ac signals during the differentiation from human embryonic stem 
cells (hESCs) to primitive cardiomyocytes (PCM). Our analysis identified three types of SEs with distinct temporal patterns (Fig. 1b-d): (a) conserved (Con, Fig. 1b), in which H3K27ac peaks are persistent throughout differentiation (e.g., HIST2 gene cluster, Fig. 1d); (b) temporally hierarchical (TH, Fig. 1b), in which a SE contains at least one H3K27ac peak that is established at the early stage and other H3K27ac peaks that are gained gradually during the differentiation course (e.g., ATP11A, Fig. 1d); and (c) de novo (DN, Fig. 1b), in which constituent peaks gain high H3K27ac signals simultaneously at late stages (e.g., $L D B 3$, Fig. 1d). On average, the H3K27ac intensities of the three types of SEs exhibit distinct temporal dynamics during differentiation (Fig. 1c), where Con and TH SEs show a relatively stable or moderate increasing profile and DN SEs show steep increases. As exemplified in Fig. 1e, the expression levels of the genes associated with the three types of SEs show a dynamic pattern that is concordant with enhancer activities. In summary, using the cardiomyocyte differentiation of hESCs as an example, we found that SEs in the differentiated cells show distinct temporal dynamics and applied a simple clustering approach to identify their temporal subtypes.

\section{SEs with different temporal patterns are associated with distinct properties and} biological functions

To investigate whether our temporal-based SE classification is functionally relevant, we performed functional enrichment analysis by using gene ontology (GO) analysis [18]. Briefly, all expressed genes within 100kb of a SE were deemed as the potential target genes. To identify the SEs' target genes of high confidence, we further used the correlation between H3K27ac signal intensity and gene expression to filter the gene set, an approach that is also used by previous studies ([19], see the "Methods" section for details). We found that the three types of SEs exhibit distinct properties and biological functions. DN SEs are enriched in biological functions highly specific to cardiomyocytes, such as "striated muscle cell differentiation" and "cardiac muscle cell development" ( $P<1 \mathrm{e}-15$, hypergeometric test), while Con and TH SEs are enriched in more general functions (e.g. "regulation of cellular biosynthetic process" and "regulation of RNA metabolic process" for conserved SEs and "post-transcriptional regulation of gene expression" and "regulation of cellular amid metabolic process" for TH SEs) $(P<1 \mathrm{e}-5$, hypergeometric test, Fig. 2a). Importantly, although Con and TH SEs are enriched in fewer specific functions than DN SEs, only 7 29\% of the Con and TH SEs are characterized with conserved activity when comparing with SEs identified from other cell types (Fig. 2b). Furthermore, most of their associated genes (70\%, 66\%, and 78\% for Con, TH and DN SEs, respectively, Additional file 1: Figure S2a) are not house-keeping genes [20].

We hypothesized that the temporal differences between the three types of SEs may reflect distinct regulatory roles at different developmental stages. Therefore, we compared the temporal expression patterns of their target genes and observed striking differences. On average, DN SE-associated genes undergo the most significant changes in transcriptional activities during differentiation, whereas Con and TH SE-associated genes are upregulated at a more moderate level (Fig. 2c, d). Moreover, DN SEassociated genes are more specifically expressed to the final stage (Fig. 2e). Consistent with this general trend, cardiomyocyte marker genes, such as MYH7 and ACTN2, are 


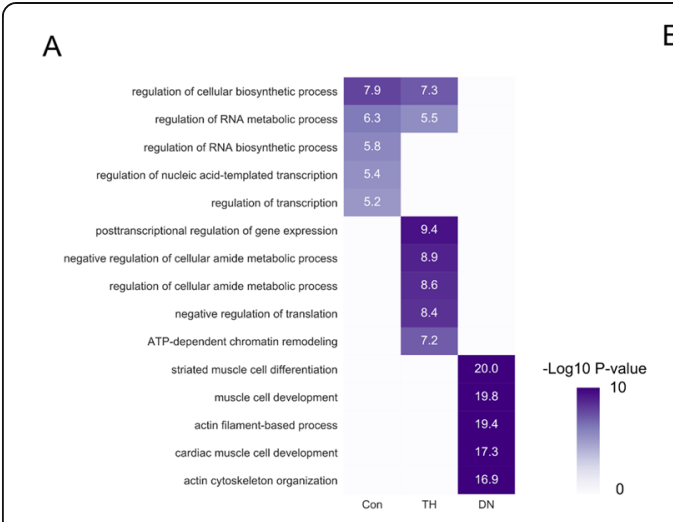

C

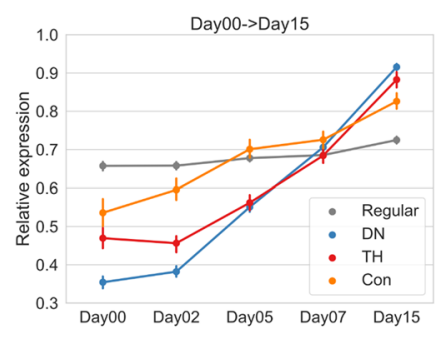

$\mathrm{F}$
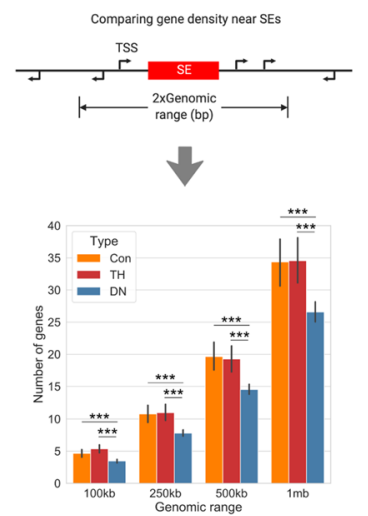

B

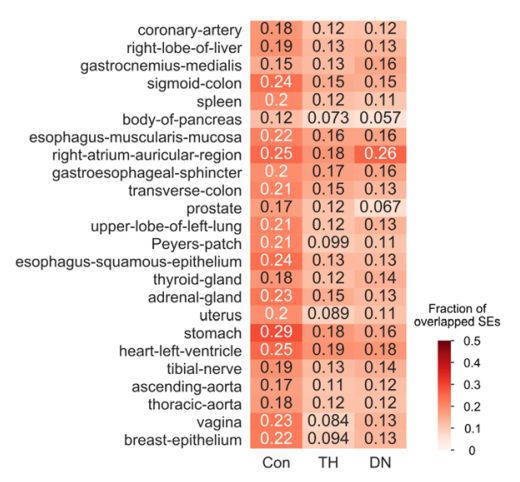

D

E

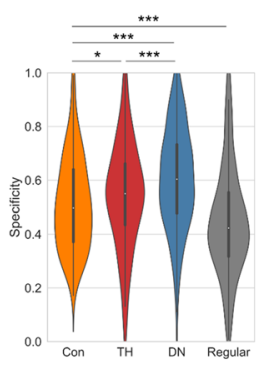

Fig. 2 Characterizations of the three types of SEs. a GO enrichment pattern of the three types of SEs. Top 5 GO terms for each type of SEs are shown after removing redundant terms. b Heatmap showing the overlap between the three types of SEs identified in human cardiomyocytes with SEs in the other 24 tissue types. Numbers in the heatmap stand for the percentage of SEs overlapped with the 24 tissue types. $\mathbf{c}$ Line plot showing the relative expression profiles for genes associated with each type of SEs. Each gene is normalized to its maximum value during differentiation and the average is shown. $\mathbf{d}$, e Comparison of the gene expression fold changes (d) and specificity (e) for the three types of SEs. Fold change is defined as the gene expression in the last stage (day 15) divided by the expression level at the first stage (day 00). Specificity measures how the genes are specifically expressed on day 15 comparing to other time points (see the "Methods" section for details). ${ }^{*} P<0.05,{ }^{*} P<0.01,{ }^{* *} P<0.001$. P-values are determined by using the Mann-Whitney $U$ test. $\mathbf{f}$ Comparison of gene density near the three types of SES. ${ }^{*} P<0.05,{ }^{* *} P<0.01$, ${ }^{* * *} P<0.001$. P-values are determined by using the Mann-Whitney $U$ test

associated with DN SEs (Additional file 1: Figure S2c-e) and their expression levels display a significant increase during differentiation (Additional file 1: Figure S2b). Of note, DN SEs tend to locate in regions with lower gene densities (Fig. 2f), suggesting that 
cell-type-specific functions are more likely to be regulated by long-range chromatin interactions comparing to less-specific functions.

\section{Characterizing the three types of SEs and their dynamics by chromatin architecture features}

The changes in transcriptional regulation landscape during cell differentiation are usually coupled with dynamic 3D chromatin organization, whereas how 3D genome organization correlates with the establishment of SEs remains poorly understood [21, 22]. Therefore, we were motivated to investigate whether the evolving landscape of SEs is associated with the 3D chromatin organization dynamics. To this end, we analyzed the accompanying $\mathrm{Hi}-\mathrm{C}$ data for cardiomyocyte differentiation [17] and quantified the dynamic changes of several well-characterized higher-order chromatin features in the following.

First, on the broadest scale, the chromatin is divided into two distinct types of compartments: A and B [23]. The A compartments are typically open and enriched with protein-coding genes, whereas the B compartments are closed and gene-poor [23]. During differentiation, switching from B to A compartments provides a mechanism for mediating the transcriptional levels of important developmental or lineage-specific genes $[21,24,25]$. To investigate to what extent compartment switching contributes to the activities of different types of SEs, we divided the genome into four types: stable A or B, dynamic compartments "A to B," and "B to A" by comparing the Hi-C compartment signals between the first and last stage of differentiation. While most SEs are located in stable A compartments (Additional file 1: Figure S3a), we also observed subtle differences among the three subtypes. DN SEs show a slightly higher percentage located in dynamic compartments ("B to A" or "A to B") than Con and TH SEs, although the differences are not statistically significant $(P=0.11$ for $\mathrm{DN}$ vs. TH SEs; $P=0.17$ for $\mathrm{DN}$ vs. Con SEs, chi-square test, Additional file 1: Figure S3a). For example, two DN SEs controlling lineage-specific genes, ACTN2 (encoding an alpha-actinin isoform that is expressed in cardiac muscles [26]) and RYR2 (encoding the RYR2 protein that functions as the major component of calcium channel that supplies ions to the cardiac muscle [27]), undergo dynamic compartment switching during cell differentiation (Fig. 3d).

Topologically-associating domains (TADs), defined as the genomic regions with high intra-domain interactions and few inter-domain interactions, act as structural units in genome organization and transcriptional regulation [28]. We then inspected the genomic position of the three types of SEs with respect to TAD boundaries. Using randomly sampled regular enhancers as the control, we found that Con and TH SEs are enriched at the boundaries of TADs (Fig. 3a, see the "Methods" section for details). Consistent with previous studies [29,30], this analysis suggests that TAD boundaries are enriched in SEs with constitutive activity.

Previous studies from our and other groups have reported that enhancers with high local chromatin interactions, such as hub enhancers [7] and frequently interacting regions (FIREs) [31, 32], play a profound role in gene regulation. FIREs are chromatin regions that show significantly high enrichment on local chromatin contacts. Genes associated with SEs associated with higher FIRE scores usually have higher expressions (Additional file 1: Figure S3b). As such, we next asked whether the three types of SEs 


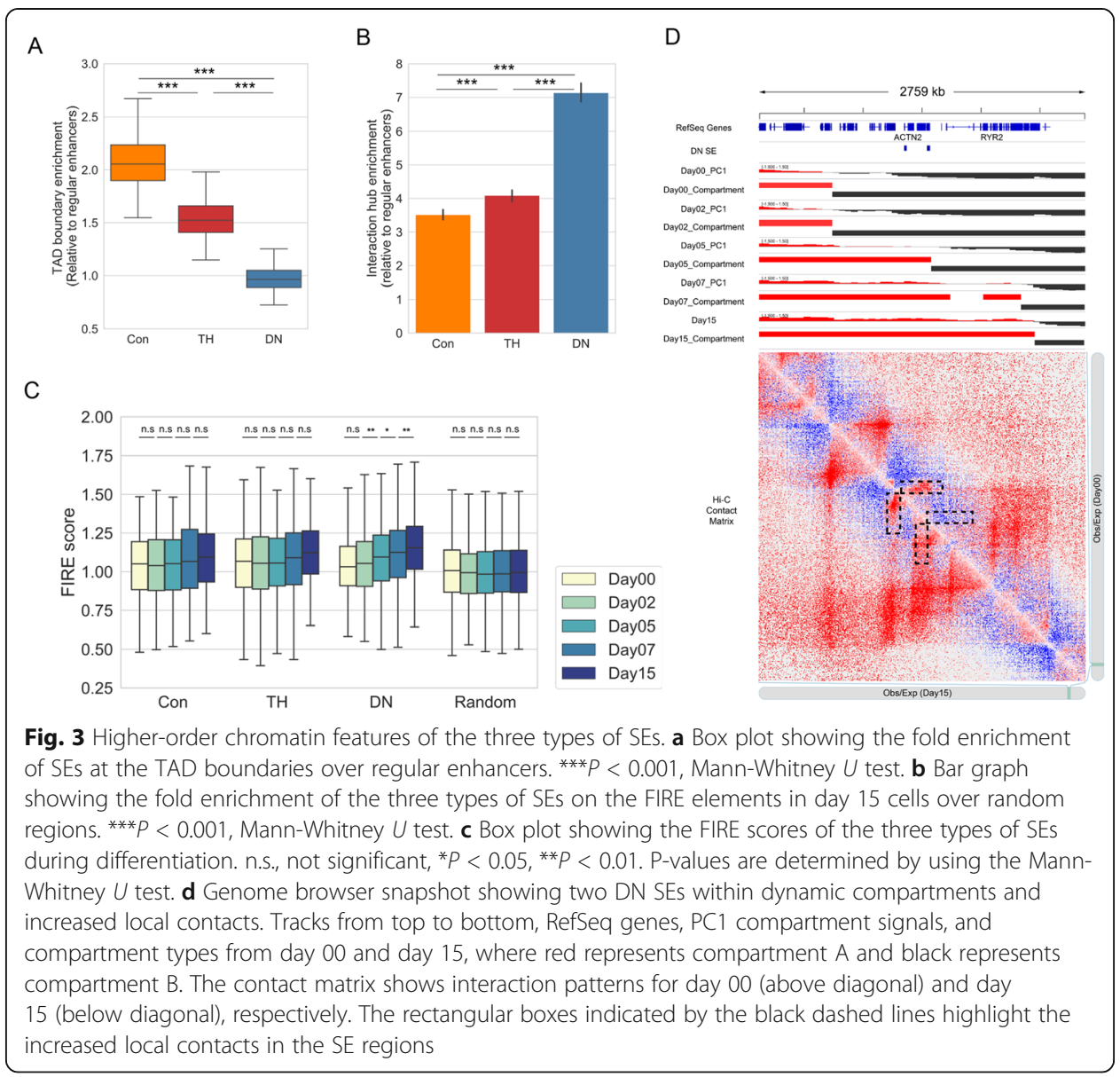

are differentially enriched in local chromatin interactions. To this end, we first identified FIREs at day15 cells by using FIREcaller [31] and tested the overlap between SEs and FIREs. DN SEs are more enriched with FIREs than Con and TH SEs, while all three types of SEs show significant enrichment in FIREs compared to random genomic regions (Fig. 3b). To check the dynamics of chromatin interaction at the three types of SEs, we then compared the FIRE scores at the three types of SEs during differentiation. As shown in Fig. 3c, DN SEs exhibit a more variable pattern in FIRE score during differentiation than Con and TH SEs. Despite the mild changes of FIRE scores between two neighboring stages, Con and TH SEs have increased spatial interactions between day 15 and day 0 , suggesting that the local interactions contribute to the stage-specific gene regulation during differentiation. As exemplified in Fig. 3d, two DN SEs located in dynamic compartments are associated with a concomitant increase of the local enrichment of $\mathrm{Hi}-\mathrm{C}$ interactions. Altogether, these results demonstrate that the dynamic chromatin conformation and local chromatin states work in concert to turn on lineage-specific genes.

\section{SE temporal classification is reproduced in other systems}

We then asked whether the patterns described above were a unique phenomenon for human cardiomyocyte differentiation or generally applicable to other differentiation 
processes or species. To this end, we repeated this procedure to analyze several public datasets.

First, we considered a public H3K27ac ChIP-seq dataset for mouse cardiomyocyte differentiation [33], which covers four differentiation stages: embryonic stem cells (ESCs), mesoderm (MES), cardiac precursor (CP) cells, and cardiomyocytes (CM). We identified three types of SEs with similar temporal patterns, which were annotated again as Con, TH, and DN, respectively (Additional file 1: Figures S4a-b, S9a). Of note, the relative proportion of each type is similar between the two species 1 1:1.5:4.2 for mouse versus 1:1.4:4.4 for human), suggesting the temporal patterns of SE activities are evolutionary conserved. Like its human counterpart, we observed significant differences in the target gene expression patterns across these three types of SEs, with the target genes of DN SEs undergoing the most dynamic changes in transcriptional activities (Additional file 1: Figure S4c-e). Functional enrichment analysis also led to similar results, where DN SEs are enriched with cardiomyocytes related functions, such as "actin filament-based process" and "heart development" $(P<1 \mathrm{e}-10$, hypergeometric test, Additional file 1: Figure S4f). Furthermore, compared to Con and TH SEs, DN SEs are associated with lower gene density, consistent with the pattern in human cardiomyocytes (Additional file 1: Figure S4g). Altogether, these results suggest that our findings are conserved between human and mouse cardiomyocytes.

Next, we tested whether the observed patterns can be generalized to other lineages. To this end, we analyzed the data from Astiaso et al. [34], which profiled the chromatin state dynamics across multiple cell types of hematopoietic differentiation for mouse in vivo. We dissected the evolving landscape of SEs in three cell types: erythroblasts, B cells, and granulocytes (GN), respectively, and found that the SEs show similar patterns. For instance, during erythropoiesis, unbiased clustering of SEs revealed three temporal patterns: Con, TH, and DN (Fig. 4a, b, Additional file 1: Figure S9b). The three types of SEs demonstrate the same hierarchy in terms of the potential in up-regulating the expression of target genes: genes associated with DN SEs are characterized with the highest fold changes during differentiation while those associated with Con SEs show relatively stable expression (Fig. 4c-e). In concordance with the higher specificity levels of genes associated with DN SEs (Fig. 4e), GO enrichment shows that DN SEs are highly enriched in biological processes that are specific to erythroid cells, such as "erythrocyte homeostasis" and "erythrocyte differentiation" $(P<1 \mathrm{e}-5$, hypergeometric test, Fig. 4f). In parallel, DN SEs are more likely to locate in less gene-dense regions than Con and TH SEs (Fig. 4g). Consistently, the same results were obtained for both B and GN cell differentiation (Additional file 1: Figures S5 and 6). Collectively, these results suggest that the three types of evolving landscape for SEs can be generalized to the development of blood lineages.

In addition to normal differentiation, cell reprogramming is induced by forced expression of master regulators and accompanied by the re-establishment of cell-typespecific chromatin states and gene expression programs. We next asked whether the identified temporal patterns could also be applied to this TF-directed reprogramming process. To this end, we analyzed the data from Chronis et al. [35], in which mouse embryonic fibroblasts (MEF) were reprogrammed to pluripotency induced by the Yamanaka factors (Oct4, Sox2, Klf4, and cMyc). We observed similar patterns except that the endpoint now is the pluripotent state (Additional file 1: Figures S7a-e, S9e). 


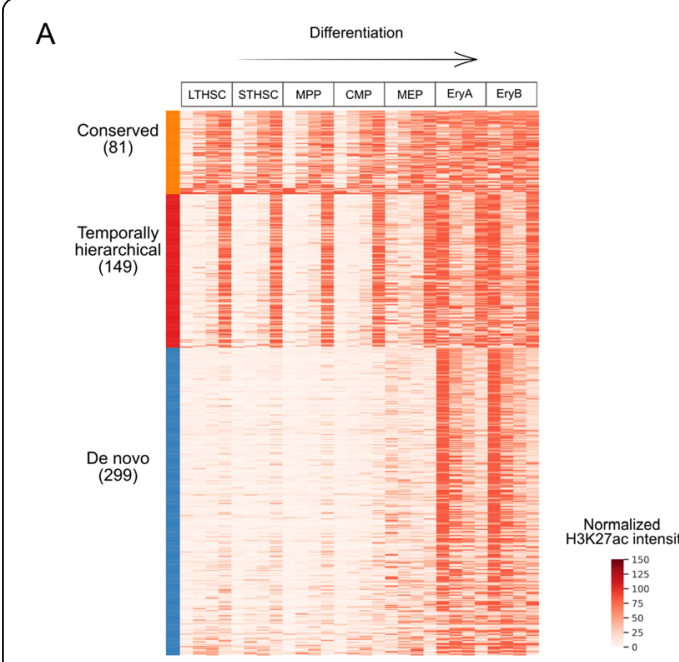

B

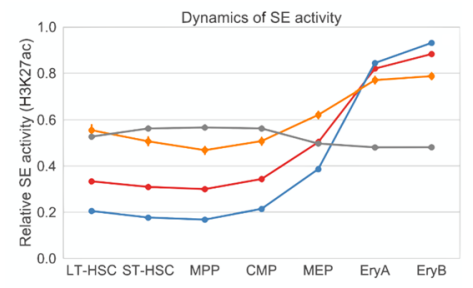

C

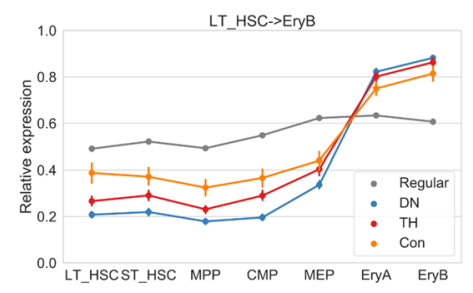

D

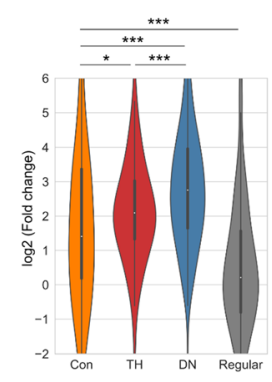

$E$

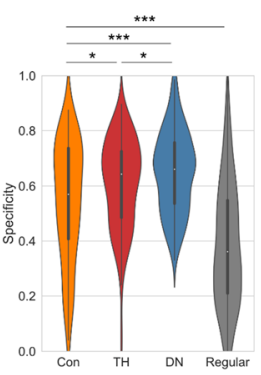

G

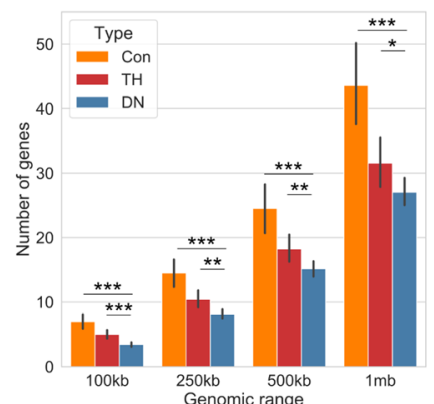

$\mathrm{F}$

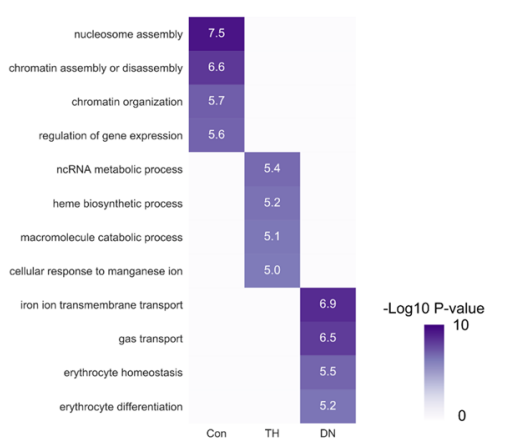

Fig. 4 Mapping the evolving landscapes of SEs in mouse erythroid cells reveals similar patterns. a K-means clustering of the SE signal vectors across erythroblast differentiation. LTHSC, long-term hematopoietic stem cells; STHSC, short-term hematopoietic stem cells; MPP, multipotent progenitor cells; CMP, common myeloid progenitor; MEP, megakaryocyte erythroid progenitor; EryA and EryB, erythroblasts. b Average dynamic profiles of the three types of SEs during differentiation. Each SE is normalized to its maximum H3K27ac intensity during differentiation and the average profile is shown. $\mathbf{c}$ Line plot showing the relative expression profiles for genes associated with each type of SEs. Each gene is normalized to its maximum value during differentiation and the average profile is shown. $\mathbf{d}$, e Comparison of the gene expression fold changes (d) and specificity (e) during differentiation for the three type of SEs. ${ }^{*}, P<0.05,{ }^{* *}, P<0.01,{ }^{* * *}, P<$ 0.001 . P-values are determined by using the Mann-Whitney $U$ test. $\mathbf{f} \mathrm{GO}$ enrichment pattern of the three types of SEs. $\mathbf{g}$ Comparison of gene density near the three types of SEs. ${ }^{*} P<0.05,{ }^{* *} P<0.01,{ }^{* * *} P<$ 0.001. P-values are determined by using the Mann-Whitney $U$ test 
Consistent with this, the functional enrichment analysis shows that DN SEs are more enriched in terms specific to ESCs, such as "multicellular organism development" and "embryo development" ( $P<1 \mathrm{e}-5$, hypergeometric test, Additional file 1: Figure S7f). The three types of SEs also exhibit differences in terms of their preferences in genomic locations, i.e., DN SEs are more likely to be located in less gene-dense regions than Con and TH SEs (Additional file 1: Figure S7g). Collectively, these results provide strong evidence that the temporal hierarchy of SE organization is generally applicable to the differentiation of diverse cell lineages and species.

\section{Decommission of SEs follows reversed temporal dynamics}

Cell differentiation is a highly dynamic process that not only requires the activation of a set of genes and enhancers specific to terminal linages, but also necessitates the decommission of genes and enhancers controlling pluripotency. Therefore, how SEs in the pluripotent cells are lost during differentiation is an intriguing question. To this end, we classified the temporal pattern of SE decommission.

We first demonstrated the characterization of SE decommissions in the human cardiomyocyte differentiation system. We identified SEs in ESCs and repeated the same procedure to classify them into different groups based on the dynamics during differentiation (Fig. 5a, Additional file 1: Figure S9f). Consistent with the SE activation analysis, the three patterns were also observed for SE decommission: conserved (Con), temporally hierarchical $(\mathrm{TH})$, and de novo $(\mathrm{DN})$, but in reverse temporal order. In contrast to Con SEs whose signals are relatively stable, DN SEs display a rapid loss of H3K27ac signals during differentiation and TH SEs demonstrate a gradual loss of H3K27ac signals (Fig. 5b). Expression levels of the genes associated with these SEs (Fig. 5c) show similar dynamics to the SE activation process. Furthermore, significant differences in the target gene expression patterns were observed for the genes associated with the three types of decommissioned SEs, with the target genes of DN SEs undergoing the most significant decrease in expression (Fig. 5c-e). GO enrichment analysis reveals that the DN SEs, which were decommissioned fast, are enriched in biological functions that are specific to ESCs, such as "multi-organism cellular process" $(P<1 \mathrm{e}-6)$ (Fig. 5f). Moreover, DN SEs in the SE decommission process are more likely to locate in less gene-dense regions than Con and TH SEs (Fig. 5g). Consistent with the results in human cardiomyocytes, the same results for the decommissioned SEs during differentiation of the erythroid cells were also obtained (Additional file 1: Figure S8, Additional file 1: Figure S9g). In summary, our results suggest that SE decommission follows reversed temporal dynamics to the SE activation.

\section{Genome editing analysis within TH SEs reveals that both early- and late-emerged enhancers are indispensable in differentiated cells}

To gain mechanistic insights into the role of temporal dynamics of SE activities during cell differentiation, we tested the effects of perturbing specific constituent elements that are activated at different stages within TH SEs on gene expression. We focused on the well-characterized in vivo mouse erythropoiesis system due to the availability for genetic manipulation. The genomic locations of the SEs and corresponding types were identified by our computational analysis described above (Fig. 4). We focused on the 


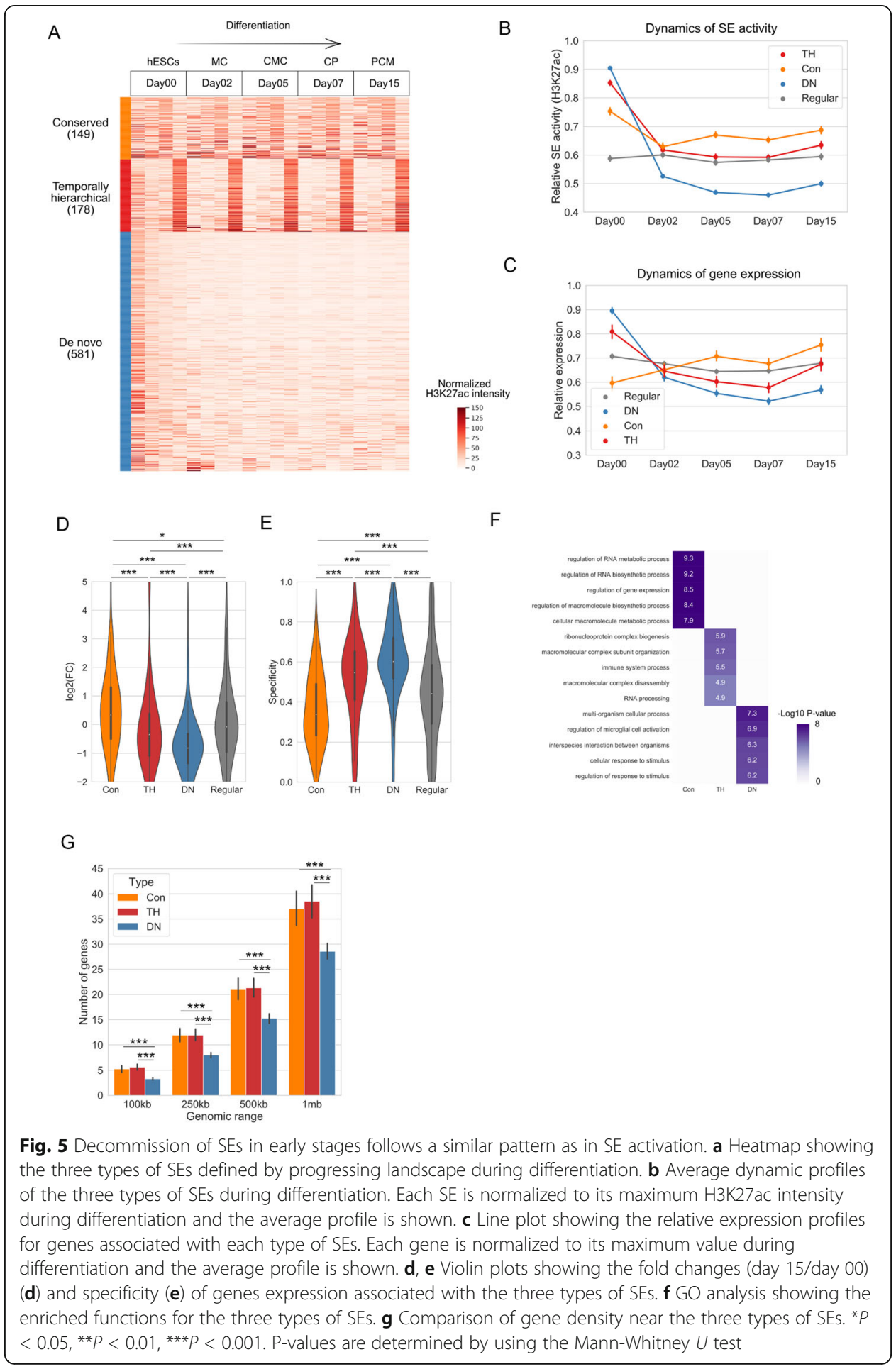

TH SEs because they contain enhancer elements that come into commission at different stages.

Based on the temporal order of enhancers' commission, we classified the enhancers within each SE into three types: early, intermediate, and late, as illustrated in Fig. 6a. Motif enrichment analysis reveals that early and late elements are enriched in distinct sets of TFs. For mouse erythroblasts, the late enhancers show a similar enrichment 


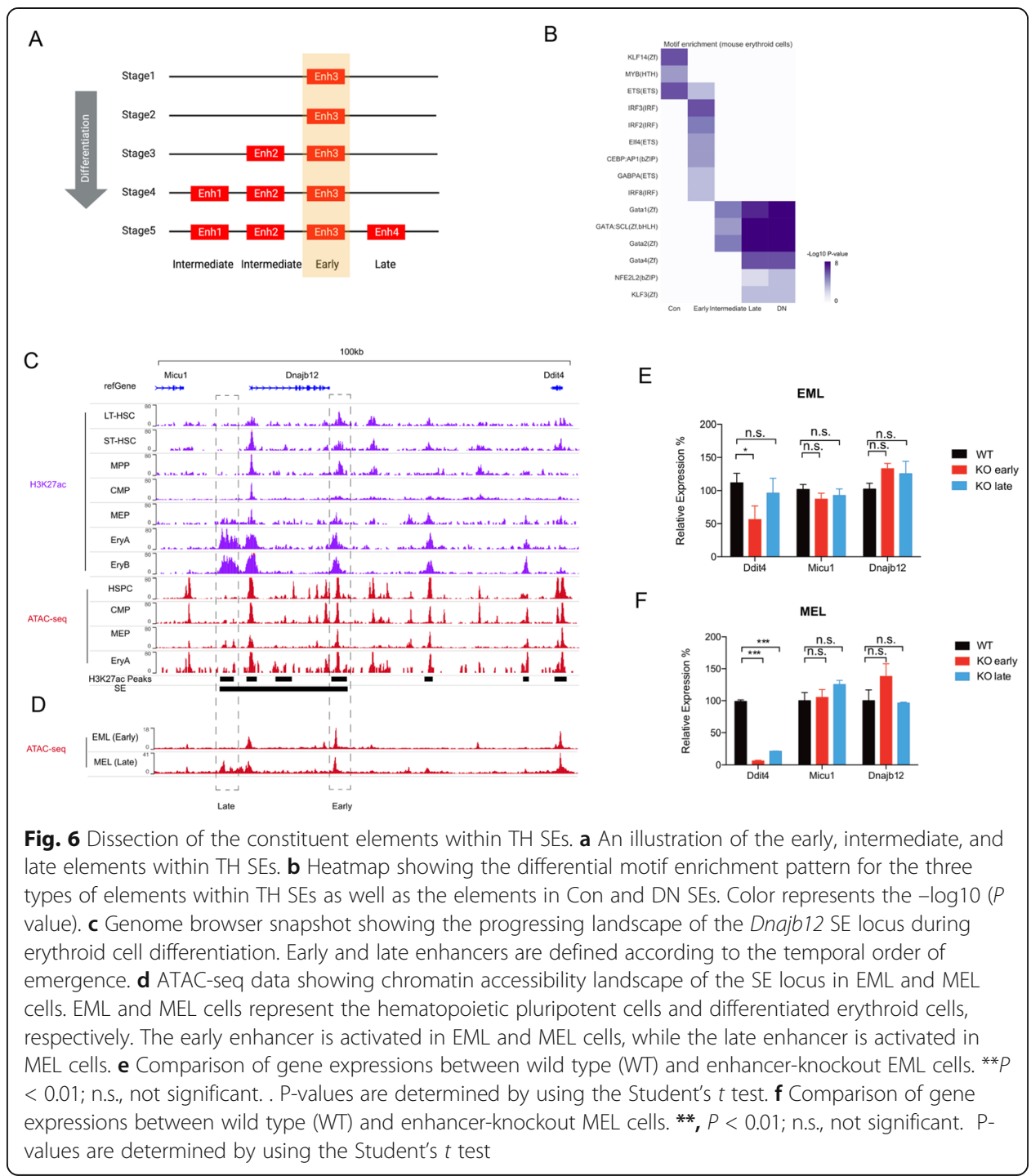

pattern with enhancers in DN SEs and are enriched in TFs with well-known functions specific to the erythroid lineage, such as Gata1 and GATA:SCL, while early enhancers are enriched with factors that are characterized with relatively general functions (e.g., ETS [36], Fig. 6b), suggesting the proper control of gene expression requires the coordinated activity of multiple factors in a temporally ordered manner. Furthermore, to test if there are factors that bind to the different enhancers in a quantitatively different way that may be omitted by motif analysis, we used Binding Analysis for Regulation of Transcription (BART) [37]. BART is a bioinformatics tool to infer functional transcription factor binding by leveraging thousands of real ChIP-seq datasets from the public domain for regulator prediction. Consistent with the motif analysis, the master regulators for erythropoiesis, such as GATA1, TAL1, and LDB1, were found to be more enriched in late enhancers than early enhancers or conserved enhancers (Additional file 1: Figure S10f). Interestingly, the architectural proteins CTCF and RAD21 also show an increasing enrichment in late enhancers (Additional file 1: Figure S10f), likely suggesting that late enhancers are more involved in regulation related to higher-order 
chromatin structure. Similarly, the differences in enriched motifs are also consistent in other lineages as well as the reprogrammed ESCs [38] (Additional file 1: Figure S10a-e). Taken together, the enrichment of distinct sets of motifs for early and late elements within TH SEs suggests that the temporal order of the establishment of elements is directed by the binding of transcription factors.

To rigorously evaluate the function of different constituent elements within TH SEs during development, we performed CRISPR-Cas9-mediated genome editing in two complementary in vitro systems: mouse erythroid-myeloid-lymphoid (EML) cells and mouse erythroleukemia (MEL) cells. EML cells, representing multipotent hematopoietic precursor cells, provide an ideal validation system in hematopoietic cells at an early stage [39], while MEL cells, immortalized erythroid cells derived from mouse adult spleens, provide a model system for mature erythroid cells at a late stage during hematopoietic differentiation $[39,40]$. We focused on the Ddit4 locus (chr10: $59,864,542-59,952,997)$, where the early enhancer in the locus was persistent during differentiation, whereas the late enhancer is erythroid-specific as H3K27ac peaks emerged at the MEP stage and are well established later in erythroblasts (Fig. 6c, d). For each system, we applied paired guide RNAs (sgRNAs) targeting $5^{\prime}$ and $3^{\prime}$ flanking regions of selected enhancers to achieve balletic editing of both enhancers respectively. The deletion size of the enhancers was refined to $1-1.5 \mathrm{~kb}$, including GATA1 motifs in the late enhancer and ETS motifs in the early enhancer. We then examined the expression levels of all nearby genes by qPCR experiments upon enhancer deletion in EML cells and MEL cells, respectively. In EML cells, Ddit4 transcripts were reduced after the KO of the early enhancer but not the late enhancer, suggesting that the early enhancer is important for regulating the target gene expression (Fig. 6e). In contrast, Ddit4 expression was remarkably reduced in the absence of either late or early enhancers in mature erythroid MEL cells, suggesting both early and late enhancers are required for gene expression at the late stage (Fig. 6f). Transcripts of Micu1 and Dnajb12 in the same locus remained unaffected upon enhancer deletion in either EML or MEL cells (Fig. 6e-f). Altogether, these results suggested that both enhancers that emerged at early and late stages are required for maintaining the proper gene expression during development, whereas late enhancers are only required for gene expression in differentiated cells.

\section{Discussion}

To dissect the evolving landscape of SEs, here we developed an unbiased computational method to characterize the temporal hierarchy of SE activity based on timecourse H3K27ac ChIP-seq data during cell differentiation. We identified three types of SEs with distinct temporal patterns, which were annotated as Con, TH, and DN, respectively. The target genes of these SEs differ in biological functions as well as transcriptional responses. In particular, the target genes of DN SEs are enriched with celltype-specific functions and exhibit strong dynamic changes of transcriptional activities. DN SEs are also associated with significant dynamic changes of 3D chromatin organizations.

SEs have been well characterized in many cell types [41-43]; however, the temporal characterization of SEs has been limited in few systems or loci. We have analyzed the genome-wide temporal establishment of SEs in multiple biological systems, including cardio-genesis, hematopoiesis, and somatic reprogramming, and across human and 
mouse lineages. Observations from those biological systems are highly consistent, suggesting the temporal patterns, Con, $\mathrm{TH}$, and $\mathrm{DN}$, are general features of SE formation and are conserved in human and mouse differentiation.

TH SEs contain a set of temporally heterogeneous regulatory elements. Those elements come into commission at different time points during differentiation and are enriched in distinct sets of TFs. Specifically, the early ones are enriched in ETS factors and late ones enriched in lineage-specific factors, such as GATA1 in erythroid cells and $\mathrm{MEF} 2 \mathrm{C}$ in cardiomyocytes, suggesting that these elements may differentially sense the external stimuli or signaling during differentiation. To dissect whether the temporal hierarchy between these enhancer elements correlates with functional hierarchy, we performed CRISPR-Cas9-based genome perturbation experiments for enhancers that emerged at early and late stages individually and found that both the early and late enhancers are indispensable to maintain the high expression of target genes in differentiated cells, whereas only early enhancers are required to maintain the expression of target genes in undifferentiated cells. Our finding is consistent with a recent study [44] that dissects the temporal and functional contributions of individual elements in the Fgf5 enhancer cluster during exit from naïve pluripotency. These pieces of evidence suggest the proper control of some genes requires the coordinated activity of multiple factors in a temporally ordered manner. Another intriguing question is whether the formation of late enhancers is dependent on the early elements. Previous studies on this aspect have led to inconsistent findings. In some cases, such as the Fgf5 enhancer cluster [44], the activities of late enhancers are not affected by the deletion of the early enhancer, whereas in other cases, such as the Wap enhancer cluster [10, 15], the early enhancer is required to activate the late enhancers. As such, the dependency among different constituent elements is locus-dependent. The computational approach developed in this paper will provide a valuable guide for future mechanistic investigations.

\section{Methods}

\section{H3K27ac peak calling and unification of peaks}

MACS1.4 [16] was used to call peaks for H3K27ac, with $P=1 \mathrm{e}-5$ as the statistical cutoff. Redundant reads were removed before peak calling. Peaks overlapped with the mm10 or hg19 blacklist ([45], https://github.com/Boyle-Lab/Blacklist/tree/master/lists) were filtered out using "bedtools intersect -v" [46].

To study enhancer dynamics across differentiation stages, we first created a peak set with unified genomic coordinates. To do this, we pooled peaks from each stage into one file and merged the overlapped peaks using "bedtools merge - $\mathrm{d} 0$," which resulted in a unified set of peaks without overlapping. Next, we compared the peaks from each individual stage with the unified peak set, and any peaks showing overlap ( $\geq 1 \mathrm{bp})$ with the peaks in the unified set were added to the stage-specific peak set using the coordinates of the unified peak. This stage-specific peak set with the coordinates of the unified peaks was used for calling SEs later.

We quantified the H3K27ac signals based on the unified peak set. Briefly, we calculated the reads per million mapped reads (RPM) for each peak at each time point, which gave a peak by time point matrix. The peak by time point matrice for all the interrogated systems were summarized in Additional file 5: Table S4. To mitigate the 
effects of differential signal-to-noise ratios among the ChIP-seq data, we then applied quantile normalization to normalize the signals across time points. The normalized matrix was used for the following clustering analysis.

\section{Identification of SEs and target genes}

SEs were identified using the ROSE algorithm [1]. Bam files of the H3K27ac ChIP-seq data after removing redundant reads and the stage-specific peak set with unified coordinates were used as input. Other parameters were set to default. We noticed that some SEs contain a high percentage of promoters; therefore, we further filtered out the SEs whose promoter peak percentage is higher than or equal to $50 \%$. A promoter peak is defined as a peak that is fully contained by the TSS $+/-2 \mathrm{~kb}$ region. By applying this criterion, a SE containing 3 or 4 peaks in total is allowed to have at most one promoter peak.

To identify the targeted genes of SEs, we took a two-step approach. First, all the expressed genes (RPKM $\geq 2$ ) within 100kb of a SE were selected as potential targets. Second, to improve the accuracy of target gene identification, we further calculated the Pearson correlation coefficient between the gene expressions and SEs' H3K27ac signals across differentiation stages, and only genes showing high correlations (Pearson correlation > 0.75) were identified as the target genes.

\section{Classification of the temporal pattern of SEs}

To compare the temporal patterns of SEs, we restricted the H3K27ac intensity matrix constructed as above to each SE, and then carried out a series of transformation steps to facilitate comparison. First, we sorted the columns according to the temporal variation of H3K27ac signals, which is quantified by the relative standard deviation (RSD) (defined as the standard deviation divided by the mean). Here, we used RSD to sort the enhancers to enable that the enhancers with similar variation/dynamics are grouped together. Since the dimension of each submatrix is different (due to the variation of the numbers of constituent elements within a $\mathrm{SE}$ ), we further standardized the number of columns to 4. Specifically, we inferred the 0th, 33.3th, 66.6th, and 100th percentiles of H3K27ac signal for each time point, termed as the four representative enhancers, and then constructed a new submatrix based on values corresponding to these percentiles. We then employed K-means clustering $(K=3)$ from scikit-learn [47] to cluster the SEs based on the four representative enhancers at different time points. 100 rounds of K-means clustering using randomly picked seed numbers were performed, and consensus clustering ([48], https://github.com/GGiecold/Cluster_Ensembles) was applied to generate a stable clustering result.

In our method, there are two parameters that could possibly impact the classification of SEs: the number of representative enhancers and the choice of $K$ in K-means clustering. To show that our method is robust to the choice of the number of representative enhancers in the clustering step, we compared the clustering result using 4 enhancers with the results using 5 or 6 enhancers. We found that the results are similar (Additional file 1: Figure S1c). Moreover, we found that as the number of cluster $\mathrm{K}$ increases, the overall pattern shown in different clusters are highly similar (Additional file 1: Figure S1d), despite that the increased number of $K$ used for clustering can reveal 
the SE dynamics in finer resolution. Therefore, our approach is robust to the choice of parameters in revealing SEs' temporal patterns.

\section{Gene expression specificity analysis}

Gene expression matrices were downloaded from GEO for each dataset involved in this study (Additional file 2: Table S1). We adopted the gene expression specificity measure, a metric developed by Xiao et al. [49] for the tissue-specific gene expression database TiSGeD, to quantify the specificity of a gene to a time point. Specifically, the expression specificity of a gene $\mathrm{G}$ at time point $\mathrm{M}$ is defined as $\sqrt{\frac{\sum_{M}^{2}}{\sum_{\text {all time points }}^{2}} X_{i}^{2}}$, where $X_{M}$ is the expression level of $\mathrm{G}$ at time point $\mathrm{M}$ and $X_{i}$ is the expression at time point $i$.

\section{House-keeping gene analysis}

House-keeping genes were downloaded from [20] and were compared with the genes associated with the three types of SEs.

\section{$\mathrm{Hi}-\mathrm{C}$ data processing and compartment identification}

Raw Hi-C data in human cardiomyocytes were downloaded from GEO with accession number GSE116862 [17]. SRA files were dumped to fastq format and then were aligned to the hg19 reference genome using HiC-Pro version 2.11.1 [50], with the default configuration parameters. A/B compartments were then identified at $50 \mathrm{~kb}$ resolution by using the "runHiCpca.pl" script from Homer [51]. For visualization, ".hic" files were downloaded from GSE116862 and visualized using Juicebox [52].

\section{Identification of TAD boundaries and enrichment of SEs at boundaries}

We used the insulation score approach by Crane et al. ([53], https://github.com/ dekkerlab/crane-nature-2015) to identify TADs based on the normalized contact matrices at $40 \mathrm{~kb}$ resolution, with $600 \mathrm{~kb}$ and $200 \mathrm{~kb}$ being used as the insulation square and delta size respectively. Bins with insulation scores higher than $0.5(\geq 0.5)$ were identified as TAD boundaries. In total, there are 3164 TAD boundaries for the day 15 sample. To test the enrichment of SE in TAD boundaries, we calculated the percentage of SE constituent enhancers overlapping with TAD boundaries for each type of SEs and compared them to the percentage of 1000 regular enhancers randomly sampled from the H3K27ac peaks. The sampling of 1000 regular enhancers was performed 20 times, and the ratio between the SE constituent enhancers and regular enhancers was calculated for plotting.

\section{Calculation of FIRE score and FIRE identification}

We used FIREcaller [31] to detect frequently interacting regions from $\mathrm{Hi}-\mathrm{C}$ data at $50 \mathrm{~kb}$ resolution. Briefly, the raw $\mathrm{Hi}-\mathrm{C}$ contact matrix was used as input and the total number of local interactions $(<200 \mathrm{~kb})$ for each genomic locus was calculated as the raw FIRE score. FIRE scores were then normalized within and across samples by FIREcaller. Within each time point, the regions with the top 10\% FIRE scores were identified as FIREs. 


\section{Motif enrichment analysis}

The "findMotifsGenome.pl" script from the HOMER package [51] was used to search for enriched motifs. The whole genome was used as the background. Factors with $P$ values lower than 1e-2 were considered as enriched. Redundant enriched motifs were merged and represented by the one with the most significant $P$ value. Enriched factors were further filtered out if the expression level of the corresponding gene is lower than 2 RPKM value throughout the differentiation.

\section{Cell culture, RNA extraction, and qRT-PCR}

EML cells were cultured in IMDM media supplemented with $20 \%$ fetal bovine serum, 2 mM L-glutamine, $1.5 \mathrm{~g} / \mathrm{L}$ sodium bicarbonate, and $100 \mathrm{ng} / \mathrm{ml}$ mouse SCF. MEL cells were cultured in a low-glucose DMEM supplemented with $10 \%$ fetal bovine serum, 2 mM L-glutamine, and Penicillin and Streptomycin. 2\% DMSO in culture medium was used to induce MEL cell differentiation. Medium was changed every other day.

The total RNA was extracted with TRIzol (Thermo Fisher) and reverse transcribed to cDNA with QuantiTect Reverse Transcription Kit (Qiagen) according to the manufacturer's instructions. cDNA samples were subjected to qRT-PCR using the iQ SYBR Green Supermix (Bio-Rad) in the CFX384 Touch Real-Time PCR Detection System (Bio-Rad). Primer sequences are listed in Additional file 4: Table S3. Values are expressed as $\log _{10} 2^{\wedge}$ DeltaCt using Actin beta (Actb) or Gapdh as a control gene.

Each transcript analysis experiment was repeated at least twice using a minimum of three biological replicates per condition. Statistical analysis was performed with an unpaired Student's $t$ test. Error bars indicate the S.E.M.; $n=3$ and 5 in Fig. 6d, e respectively. $P$ values were calculated and statistical significance indicates ${ }^{*} P<0.05$, ${ }^{* *} P<0.01$, and ${ }^{* * *} P<0.001$.

\section{CRISPR-Cas9-mediated perturbation of enhancers}

To generate biallelic deletion of the late enhancer in SE loci, sgRNAs targeting $5^{\prime}$ - and 3 '-flanking regions of the targeted enhancers were designed and synthesized respectively. sgRNA sequences are listed in Additional file 3: Table S2. Two overlapping oligonucleotides carrying sgRNA sequence targeting $5^{\prime}$-flanking region and two overlapping oligonucleotides carrying sgRNA targeting 3 '-flanking region were annealed and cloned, respectively, as previously described [54]. In brief, 10-uM guide sequence oligos and 10-uM complement oligo were mixed with $1 \mathrm{X}$ ligation buffer supplemented with 5 $\mathrm{U}$ of T4 polynucleotide kinase (PNK) in $10 \mathrm{ul}$ reaction. Anneal in a thermocycler using $37^{\circ} \mathrm{C}$ for $30 \mathrm{~min} ; 95^{\circ} \mathrm{C}$ for $5 \mathrm{~min}$ and then ramp down to $25^{\circ} \mathrm{C}$ at $0.1^{\circ} \mathrm{C} / \mathrm{s}$. The annealed oligos were then ligated into pKLV-U6gRNA(BbsI)-PGK-BFP (Addgene \#50946) vector using a Golden Gate assembly. Ligation mixture [100 ng vector, $1 \mathrm{uM}$ annealed oligos, $40 \mathrm{U}$ BbsI restriction enzyme (NEB), $1 \mathrm{mM} \mathrm{ATP}, 0.1 \mathrm{mg} / \mathrm{ml} \mathrm{BSA}$ and $750 \mathrm{U}$ T4 DNA ligase (NEB), and $1 \mathrm{X}$ restriction enzyme buffer] were incubated in a thermocycler using 20 cycles of $37^{\circ} \mathrm{C}$ for $5 \mathrm{~min}, 20^{\circ} \mathrm{C}$ for $5 \mathrm{~min}$; followed by $80^{\circ} \mathrm{C}$ for $20 \mathrm{~min}$.

pKLV-U6gRNA(BbsI)-PGK-BFP construct with sgRNA targeting $5^{\prime}$-flanking region and pKLV-U6gRNA(BbsI)-PGK-BFP construct with sgRNA targeting 3 '-flanking region were co-transfected with pCas9-GFP (Addgene \#44719) at the ratio of 1:1:2 into MEL cells by Lipofectamine 2000 (Invitrogen). The top $5 \%$ of $\mathrm{GFP}^{+} / \mathrm{BFP}^{+}$cells $48 \mathrm{~h}$ post- 
transfection were isolated by FACS. Single cell-derived colonies were screened for biallelic deletion of the targeting region.

A similar strategy with sgRNAs targeting flanking regions of selected enhancers has been applied to generate biallelic deletion of the late enhancer in Ddit4 locus, and biallelic deletion of the early enhancer in Ddit4 locus. sgRNA sequences and genotyping primer sequences are listed in Additional file 4: Table S3. Similar strategies were used to generate biallelic deletion of enhancers in EML cells with the exception that both Cas9 and sgRNA were stably expressed using lentivirus.

\section{Abbreviations}

SE: Super-enhancer; ChIP-seq: Chromatin immune-precipitation followed by high-throughput sequencing; TSS: Transcription start site; Hi-C: Genome conformation capture; TAD: Topologically associating domain; FIREs: Frequently interacting regions; RSD: Relative standard deviation; hESC: Human embryonic stem cells; mESC: Mouse embryonic stem cells; MC: Mesodermal cells; CMC: Cardiac mesodermal cells; CP: Cardiac progenitors; PCM: Primitive cardiomyocytes; CM: Cardiomyocytes; LTHSC: Long-term hematopoietic stem cells; STHSC: Short-term hematopoietic stem cells; MPP: Multipotent progenitor; CMP: Common myeloid progenitor; MEP: Megakaryocyte erythroid progenitor; CLP: Common lymphoid progenitor; GMP: Granulocyte-monocyte progenitor; GN: Granulocyte; MEFs: Mouse embryonic fibroblasts

\section{Supplementary Information}

The online version contains supplementary material available at https://doi.org/10.1186/s13059-021-02485-x.

Additional file 1. Supplementary figures (Figure S1 - Figure S10).

Additional file 2: Table S1. Summary of used datasets in the study.

Additional file 3: Table S2. List of target enhancers, guide RNAs sequences, and genotyping PCR primers.

Additional file 4: Table S3. List of qRT-PCR primers used for experimental validation.

Additional file 5: Table S4. Summary of the evolving signals of enhancers during differentiation for each interrogated system.

Additional file 6. Review History.

\section{Acknowledgements}

We thank the labs which produced the public data used in this study. We thank the useful comments from the members of SHO and GCY labs.

\section{Review history}

The review history is available as Additional file 6.

\section{Peer review information}

Tim Sands was the primary editor of this article and managed its editorial process and peer review in collaboration with the rest of the editorial team.

\section{Authors' contributions}

YK, WC, JH, and GCY conceived and designed the study. YK performed the computational analyses. BEL, MZ, GYL, FC, $\mathrm{YH}$, and WC performed the enhancer perturbation experiments. HJC generated the H3K27ac ChIP-seq data in MEL cells. YK, WC, JH, and GCY wrote the manuscript with the contributions from all authors. GCY, JH, and WC and SHO supervised the study. The authors read and approved the final manuscript.

\section{Authors' information}

Yan Kai, Bin E. Li and Ming Zhu are co-first authors. Guo-Cheng Yuan, Jialiang Huang, and Wenqing Cai are co-senior authors.

\section{Funding}

This work was supported by the NIH Cooperative Centers of Excellence in Hematology award (5U54DK11805) to SHO, grants (R01HG009663) to GCY, and National Natural Science Foundation of China (31871317, 32070635) and Natural Science Foundation of Fujian Province of China (2020J01028) to JH.

\section{Availability of data and materials}

The data of the human cardiomyocyte differentiation system were downloaded from the study by Zhang et al. [17] with accession number GSE1 16862 [55]. Mouse cardiomyocyte data were downloaded from Wamstad et al. [33] with accession number GSE47950 [56]. The data of the mouse erythroid, B and granulocytes were downloaded from LaraAstiaso et al. with accession number GSE60103 [57]. The reprogramming data in mouse were downloaded from Constantinos Chronis et al. with accession number GSE90895 [58]. SEs of the compendium of tissue types used in this study were downloaded from Jiang et al. SEdb: a comprehensive human super-enhancer database (http://www. licpathway.net/sedb) [59]. A list of all used datasets and accession numbers were summarized in Additional file 2: Table 
S1. The evolving signals of enhancers during cell differentiation for all the interrogated systems in this study are summarized in Additional file 5: Table S4.

\section{Declarations}

\section{Ethics approval and consent to participate}

Not applicable.

\section{Consent for publication}

Not applicable.

\section{Competing interests}

The authors declare no competing interests.

\section{Author details}

${ }^{1}$ Department of Pediatric Oncology, Dana-Farber Cancer Institute and Harvard Medical School, Boston, MA 02115, USA. ${ }^{2}$ Cancer and Blood Disorders Center, Boston Children's Hospital and Dana-Farber Cancer Institute, Harvard Medical School, Boston, MA 02115, USA. ${ }^{3}$ State Key Laboratory of Cellular Stress Biology, Innovation Center for Cell Signaling Network, School of Life Sciences, Xiamen University, Xiamen 361102, Fujian, China. ${ }^{4}$ Howard Hughes Medical Institute, Boston, MA 02115, USA. ${ }^{5}$ Department of Genetics and Genomic Sciences, Charles Bronfman Institute for Precision Medicine, Icahn School of Medicine at Mount Sinai, New York, NY 10029, USA.

Received: 11 June 2020 Accepted: 2 September 2021

Published online: 15 September 2021

\section{References}

1. Whyte WA, Orlando DA, Hnisz D, Abraham BJ, Lin CY, Kagey MH, et al. Master transcription factors and mediator establish super-enhancers at key cell identity genes. Cell. 2013;153(2):307-19. https://doi.org/10.1016/j.cell.2013.03.035.

2. Parker SC, Stitzel ML, Taylor DL, Orozco JM, Erdos MR, Akiyama JA, et al. Chromatin stretch enhancer states drive cellspecific gene regulation and harbor human disease risk variants. Proc Natl Acad Sci U S A. 2013;110(44):17921-6. https:// doi.org/10.1073/pnas.1317023110.

3. Hnisz D, Schuijers J, Lin CY, Weintraub AS, Abraham BJ, Lee TI, et al. Convergence of developmental and oncogenic signaling pathways at transcriptional super-enhancers. Mol Cell. 2015;58(2):362-70. https://doi.org/10.1016/j.molcel.2015. 02.014.

4. Hnisz D, Abraham BJ, Lee TI, Lau A, Saint-Andre V, Sigova AA, et al. Super-enhancers in the control of cell identity and disease. Cell. 2013;155(4):934-47. https://doi.org/10.1016/j.cell.2013.09.053.

5. Wang X, Cairns MJ, Yan J. Super-enhancers in transcriptional regulation and genome organization. Nucleic Acids Res. 2019:47(22):11481-96. https://doi.org/10.1093/nar/gkz1038.

6. Huang J, Liu X, Li D, Shao Z, Cao H, Zhang Y, et al. Dynamic control of enhancer repertoires drives lineage and stagespecific transcription during Hematopoiesis. Dev Cell. 2016;36(1):9-23. https://doi.org/10.1016/j.devcel.2015.12.014.

7. Huang J, Li K, Cai W, Liu X, Zhang Y, Orkin SH, et al. Dissecting super-enhancer hierarchy based on chromatin interactions. Nat Commun. 2018;9(1):943. https://doi.org/10.1038/s41467-018-03279-9.

8. Liu X, Chen Y, Zhang Y, Liu Y, Liu N, Botten GA, et al. Multiplexed capture of spatial configuration and temporal dynamics of locus-specific 3D chromatin by biotinylated dCas9. Genome Biol. 2020;21(1):59. https://doi.org/10.1186/s13 059-020-01973-w.

9. Bahr C, von Paleske L, Uslu W, Remeseiro S, Takayama N, Ng SW, et al. A Myc enhancer cluster regulates normal and leukaemic haematopoietic stem cell hierarchies. Nature. 2018:553(7689):515-20. https://doi.org/10.1038/nature25193.

10. Shin HY, Willi M, HyunYoo K, Zeng X, Wang C, Metser G, et al. Hierarchy within the mammary STAT5-driven Wap superenhancer. Nat Genet. 2016;48(8):904-11. https://doi.org/10.1038/ng.3606.

11. Brown JD, Lin CY, Duan Q, Griffin G, Federation A, Paranal RM, et al. NF-kappaB directs dynamic super enhancer formation in inflammation and atherogenesis. Mol Cell. 2014;56(2):219-31. https://doi.org/10.1016/j.molcel.2014.08.024.

12. Hah N, Benner C, Chong LW, Yu RT, Downes M, Evans RM. Inflammation-sensitive super enhancers form domains of coordinately regulated enhancer RNAs. Proc Natl Acad Sci U S A. 2015;112(3):E297-302. https://doi.org/10.1073/pna S.1424028112.

13. Kitagawa Y, Ohkura N, Kidani Y, Vandenbon A, Hirota K, Kawakami R, et al. Guidance of regulatory T cell development by Satb1-dependent super-enhancer establishment. Nat Immunol. 2017;18(2):173-83. https://doi.org/10.1038/ni.3646.

14. Bojcsuk D, Nagy G, Balint BL. Inducible super-enhancers are organized based on canonical signal-specific transcription factor binding elements. Nucleic Acids Res. 2017:45:3693-706. https://doi.org/10.1093/nar/gkw1283.

15. Lee HK, Willi M, Shin HY, Liu C, Hennighausen L. Progressing super-enhancer landscape during mammary differentiation controls tissue-specific gene regulation. Nucleic Acids Res. 2018;46(20):10796-809. https://doi.org/10.1093/nar/gky891.

16. Zhang Y, Liu T, Meyer CA, Eeckhoute J, Johnson DS, Bernstein BE, et al. Model-based analysis of ChIP-Seq (MACS). Genome Biol. 2008;9(9):R137. https://doi.org/10.1186/gb-2008-9-9-r137.

17. Zhang Y, Li T, Preiss S, Amaral ML, Grinstein JD, Farah EN, et al. Transcriptionally active HERV-H retrotransposons demarcate topologically associating domains in human pluripotent stem cells. Nat Genet. 2019;51(9):1380-8. https://doi. org/10.1038/s41588-019-0479-7.

18. McLean CY, Bristor D, Hiller M, Clarke SL, Schaar BT, Lowe CB, et al. GREAT improves functional interpretation of cisregulatory regions. Nat Biotechnol. 2010;28(5):495-501. https://doi.org/10.1038/nbt.1630.

19. Gerard D, Schmidt F, Ginolhac A, Schmitz M, Halder R, Ebert P, et al. Temporal enhancer profiling of parallel lineages identifies AHR and GLIS1 as regulators of mesenchymal multipotency. Nucleic Acids Res. 2019:47(3):1141-63. https://doi. org/10.1093/nar/gky1240. 
20. Eisenberg E, Levanon EY. Human housekeeping genes, revisited. Trends Genet. 2013;29(10):569-74. https://doi.org/10.1 016/j.tig.2013.05.010.

21. Zheng $\mathrm{H}$, Xie W. The role of 3D genome organization in development and cell differentiation. Nat Rev Mol Cell Biol. 2019;20(9):535-50. https://doi.org/10.1038/541580-019-0132-4.

22. Beagrie RA, Scialdone A, Schueler M, Kraemer DC, Chotalia M, Xie SQ, et al. Complex multi-enhancer contacts captured by genome architecture mapping. Nature. 2017;543(7646):519-24. https://doi.org/10.1038/nature21411.

23. Lieberman-Aiden E, van Berkum NL, Williams L, Imakaev M, Ragoczy T, Telling A, et al. Comprehensive mapping of longrange interactions reveals folding principles of the human genome. Science. 2009;326(5950):289-93. https://doi.org/1 $0.1126 /$ science. 1181369

24. Dixon JR, Jung I, Selvaraj S, Shen Y, Antosiewicz-Bourget JE, Lee AY, et al. Chromatin architecture reorganization during stem cell differentiation. Nature. 2015;518(7539):331-6. https://doi.org/10.1038/nature14222.

25. Takebayashi S, Dileep V, Ryba T, Dennis JH, Gilbert DM. Chromatin-interaction compartment switch at developmentally regulated chromosomal domains reveals an unusual principle of chromatin folding. Proc Natl Acad Sci U S A. 2012; 109(31):12574-9. https://doi.org/10.1073/pnas.1207185109.

26. Bagnall RD, Molloy LK, Kalman JM, Semsarian C. Exome sequencing identifies a mutation in the ACTN2 gene in a family with idiopathic ventricular fibrillation, left ventricular noncompaction, and sudden death. BMC Med Genet. 2014;15(1):99. https://doi.org/10.1186/s12881-014-0099-0.

27. Otsu K, Willard HF, Khanna VK, Zorzato F, Green NM, MacLennan DH. Molecular cloning of CDNA encoding the Ca2+ release channel (ryanodine receptor) of rabbit cardiac muscle sarcoplasmic reticulum. J Biol Chem. 1990;265(23):1347283. https://doi.org/10.1016/S0021-9258(18)77371-7.

28. Dixon JR, Selvaraj S, Yue F, Kim A, Li Y, Shen Y, et al. Topological domains in mammalian genomes identified by analysis of chromatin interactions. Nature. 2012;485(7398):376-80. https://doi.org/10.1038/nature11082.

29. Ulianov SV, Khrameeva EE, Gavrilov AA, Flyamer IM, Kos P, Mikhaleva EA, et al. Active chromatin and transcription play a key role in chromosome partitioning into topologically associating domains. Genome Res. 2016;26(1):70-84. https://doi. org/10.1101/gr.196006.115.

30. Szabo Q, Bantignies F, Cavalli G. Principles of genome folding into topologically associating domains. Sci Adv. 2019;5: eaaw1668.

31. Crowley C, Yang Y, Qiu Y, Hu B, Abnousi A, Lipinski J, et al. FIREcaller: detecting frequently interacting regions from Hi-C data. Comput Struct Biotechnol J. 2021;19:355-62. https://doi.org/10.1016/j.csbj.2020.12.026.

32. Schmitt AD, Hu M, Jung I, Xu Z, Qiu Y, Tan CL, et al. A compendium of chromatin contact maps reveals spatially active regions in the human genome. Cell Rep. 2016;17(8):2042-59. https://doi.org/10.1016/j.celrep.2016.10.061.

33. Wamstad JA, Alexander JM, Truty RM, Shrikumar A, Li F, Eilertson KE, et al. Dynamic and coordinated epigenetic regulation of developmental transitions in the cardiac lineage. Cell. 2012;151(1):206-20. https://doi.org/10.1016/j.cell.2 012.07.035.

34. Lara-Astiaso D, Weiner A, Lorenzo-Vivas E, Zaretsky I, Jaitin DA, David E, et al. Immunogenetics. Chromatin state dynamics during blood formation. Science. 2014;345(6199):943-9. https://doi.org/10.1126/science.1256271.

35. Chronis C, Fiziev P, Papp B, Butz S, Bonora G, Sabri S, et al. Cooperative binding of transcription factors orchestrates reprogramming. Cell. 2017;168(3):442-459 e420. https://doi.org/10.1016/j.cell.2016.12.016.

36. Curina A, Termanini A, Barozzi I, Prosperini E, Simonatto M, Polletti S, et al. High constitutive activity of a broad panel of housekeeping and tissue-specific cis-regulatory elements depends on a subset of ETS proteins. Genes Dev. 2017;31(4): 399-412. https://doi.org/10.1101/gad.293134.116.

37. Wang Z, Civelek M, Miller CL, Sheffield NC, Guertin MJ, Zang C. BART: a transcription factor prediction tool with query gene sets or epigenomic profiles. Bioinformatics. 2018;34(16):2867-9. https://doi.org/10.1093/bioinformatics/bty194.

38. David L, Polo JM. Phases of reprogramming. Stem Cell Res. 2014;12(3):754-61. https://doi.org/10.1016/.jscr.2014.03.007.

39. Ganguly S, Skoultchi Al. Absolute rates of globin gene transcription and mRNA formation during differentiation of cultured mouse erythroleukemia cells. J Biol Chem. 1985;260(22):12167-73. https://doi.org/10.1016/S0021-9258(17)390026.

40. Sheffery M, Marks PA, Rifkind RA. Gene expression in murine erythroleukemia cells. Transcriptional control and chromatin structure of the alpha 1-globin gene. J Mol Biol. 1984;172(4):417-36. https://doi.org/10.1016/S0022-2836(84 80015-7.

41. Kai Y, Andricovich J, Zeng Z, Zhu J, Tzatsos A, Peng W. Predicting CTCF-mediated chromatin interactions by integrating genomic and epigenomic features. Nat Commun. 2018;9(1):4221. https://doi.org/10.1038/s41467-018-06664-6.

42. Jiang Y, Qian F, Bai X, Liu Y, Wang Q, Ai B, et al. SEdb: a comprehensive human super-enhancer database. Nucleic Acids Res. 2019;47(D1):D235-43. https://doi.org/10.1093/nar/gky1025

43. Ryu J, Kim H, Yang D, Lee AJ, Jung I. A new class of constitutively active super-enhancers is associated with fast recovery of 3D chromatin loops. BMC Bioinformatics. 2019;20(S3):127. https://doi.org/10.1186/s12859-019-2646-3.

44. Thomas HF, Kotova E, Jayaram S, Pilz A, Romeike M, Lackner A, et al. Temporal dissection of an enhancer cluster reveals distinct temporal and functional contributions of individual elements. Mol Cell. 2021;81(5):969-82 e913. https://doi.org/10.1016/.j.molcel.2020.12.047.

45. Amemiya HM, Kundaje A, Boyle AP. The ENCODE blacklist: identification of problematic regions of the genome. Sci Rep. 2019;9(1):9354. https://doi.org/10.1038/s41598-019-45839-z.

46. Quinlan AR, Hall IM. BEDTools: a flexible suite of utilities for comparing genomic features. Bioinformatics. 2010;26(6):8412. https://doi.org/10.1093/bioinformatics/btq033.

47. Abraham A, Pedregosa F, Eickenberg M, Gervais P, Mueller A, Kossaifi J, et al. Machine learning for neuroimaging with scikit-learn. Front Neuroinform. 2014;8:14.

48. Giecold G, Marco E, Garcia SP, Trippa L, Yuan GC. Robust lineage reconstruction from high-dimensional single-cell data. Nucleic Acids Res. 2016;44(14):e122. https://doi.org/10.1093/nar/gkw452.

49. Xiao SJ, Zhang C, Zou Q, Ji ZL. TiSGeD: a database for tissue-specific genes. Bioinformatics. 2010;26(9):1273-5. https:// doi.org/10.1093/bioinformatics/btq109.

50. Servant N, Varoquaux N, Lajoie BR, Viara E, Chen CJ, Vert JP, et al. HiC-Pro: an optimized and flexible pipeline for Hi-C data processing. Genome Biol. 2015;16(1):259. https://doi.org/10.1186/s13059-015-0831-x. 
51. Heinz S, Benner C, Spann N, Bertolino E, Lin YC, Laslo P, et al. Simple combinations of lineage-determining transcription factors prime cis-regulatory elements required for macrophage and B cell identities. Mol Cell. 2010;38(4):576-89. https:// doi.org/10.1016/j.molcel.2010.05.004

52. Durand NC, Robinson JT, Shamim MS, Machol I, Mesirov JP, Lander ES, et al. Juicebox provides a visualization system for Hi-C contact maps with unlimited zoom. Cell Syst. 2016;3(1):99-101. https://doi.org/10.1016/j.cels.2015.07.012.

53. Crane E, Bian Q, McCord RP, Lajoie BR, Wheeler BS, Ralston EJ, et al. Condensin-driven remodelling of X chromosome topology during dosage compensation. Nature. 2015;523(7559):240-4. https://doi.org/10.1038/nature14450.

54. Cai W, Huang J, Zhu Q, Li BE, Seruggia D, Zhou P, et al. Enhancer dependence of cell-type-specific gene expression increases with developmental age. Proc Natl Acad Sci U S A. 2020;117(35):21450-8. https://doi.org/10.1073/pnas.2 008672117.

55. Zhang Y, Li T, Preiss S, Amaral ML, Grinstein JD, Farah EN, et al. Transcriptionally active HERV-H retrotransposons demarcate topologically associating domains in human pluripotent stem cells. Gene Expr Omnibus. 2019. https://www. ncbi.nIm.nih.gov/geo/query/acc.cgi?acc=GSE116862;51(9):1380-8. https://doi.org/10.1038/s41588-019-0479-7.

56. Wamstad JA, Alexander JM, Truty RM, Shrikumar A, Li F, Eilertson KE, et al. Dynamic and coordinated epigenetic regulation of developmental transitions in the cardiac lineage. Gene Expr Omnibus. 2013; https://www.ncbi.nlm.nih.gov/ geo/query/acc.cgi?acc=GSE116862.

57. Lara-Astiaso D, Weiner A, Lorenzo-Vivas E, Zaretsky I, Jaitin DA, David E, et al. Immunogenetics. Chromatin state dynamics during blood formation. Gene Expr Omnibus. 2014; https://www.ncbin.nlm.nih.gov/geo/query/acc.cgi?acc= GSE60103.

58. Chronis C, Fiziev P, Papp B, Butz S, Bonora G, Sabri S, et al. Cooperative binding of transcription factors orchestrates reprogramming. Gene Expr Omnibus. 2017. https://www.ncbi.nlm.nih.gov/geo/query/acc.cgi?acc=GSE90895;168(3):442459.e20. https://doi.org/10.1016/j.cell.2016.12.016.

59. Jiang Y, Qian F, Bai X, Liu Y, Wang Q, Ai B, Han X, Shi S, Zhang J, Li X, et al. SEdb: a comprehensive human superenhancer database. SEdb. http://www.licpathway.net/sedb/. (Accessed $1^{\text {st }}$ Sept 2021).

\section{Publisher's Note}

Springer Nature remains neutral with regard to jurisdictional claims in published maps and institutional affiliations.

\section{Ready to submit your research? Choose BMC and benefit from:}

- fast, convenient online submission

- thorough peer review by experienced researchers in your field

- rapid publication on acceptance

- support for research data, including large and complex data types

- gold Open Access which fosters wider collaboration and increased citations

- maximum visibility for your research: over $100 \mathrm{M}$ website views per year

At $\mathrm{BMC}$, research is always in progress. 